\title{
PROJECTIVE MODELING AND SYSTEM CHANGE: RESERVOIR MANAGEMENT EXAMPLES
}

\author{
K.J. Keesman* \\ * Systems and Control Group, Wageningen University \\ THE NETHERLANDS \\ Tel.: +31317 483780, Fax: +31317414957 \\ E-mail address: karel.keesman@wur.nl
}

\begin{abstract}
In this paper a projective modeling approach for ecological/environmental systems is introduced. The basic idea behind projective modeling is to define (possible) future output behavior and to use identifiable timevarying system parameters, representing underlying sub-processes, as an (additional) agent for control in order to investigate potential off-normal system changes. Recursive estimation techniques are used to calculate the parameter trajectories, which subsequently, for full understanding of the problem, are subject to physical interpretation. This idea of projective modeling is motivated by a real-world eutrophication example and further explained in some more detail on a class-room example, describing the outflow from a reservoir. (CIFAC 2006
\end{abstract}

Keywords: modeling, identification, recursive approaches, environment, ecology

\section{INTRODUCTION}

In the last decades the main stream of modeling approaches was on predictive modeling. That is, modeling as a vehicle to predict system's behavior in the near or far future. The prediction-error methods (see e.g. Ljung (1987)), as a generalization of the common system identification methods that aimed at minimizing the residuals, may certainly have contributed to this. In this approach, in addition to (some) prior knowledge of the system, the availability of experimental data was a prerequisite for system identification and parameter estimation. However, the main limitation of the predictive modeling approach is that the model predictions only reflect the past system's behavior, as described by the model equations and experimental data. Physical or mechanistic modeling in its pure form, on the contrary, focuses on the deduction of models from prior knowledge only. Hence, in general, the resulting model, in particular suitable for process design, reflects the state-of-the-art of mathematical modeling of a specific process. As an alternative to these approaches, in the mid-eighties, J.C. Willems introduced a so-called behavioral approach to modeling (see e.g. Antoulas and Willems (1993) for details on identification issues).

Especially, in environmental systems modeling under expected environmental change the key question is: how does an internal system change effect the system's behavior in the future? This question cannot be answered with the existing modeling approaches. First of all no experimental data is available and, in addition to this, it is unknown how system mechanisms change in future. For this type of problem we introduce the concept of projective modeling, which is closely related to Ormell's definition of projective modeling. Ormell (1977) asserts: "... Mathematics is the science of ifs. It is concerned with the possibilities, 
rather than with the actualities, of the world. (...) Mathematics used in this way, to 'look at' the implications of a theory or a proposal, we call projective modeling."

The aim of this paper is to further describe the projective modeling approach for dynamic systems, without focusing on mathematical technicalities. Hence, in essence the philosophy behind projective modeling is exposed. The basic idea is to define (possible) future output behavior and to use identifiable time-varying system parameters as an (additional) agent for control. This idea is, especially, useful for environmental systems with (i) limited prior knowledge of the mechanisms that govern the processes, (ii) large response times, and (iii) limited options for collecting experimental data. Furthermore, and this is essential for our approach, it uses feedback unlike the commonly used error propagation studies with stochastic (see e.g Tiwari and Hobbie (1976)) or bounded parameters (see e.g Keesman and van Straten (1987), van Straten and Keesman (1991) and Kurzhanski and Varaiya (2000)). This work has been inspired by the ideas as presented in Beck (2002), in particular chapters 18 and 19 (Keesman (2002); Kryazhimskii and Beck (2002)). For educational purposes only, a very simple theoretically identifiable dynamic system will be used to illustrate the ideas.

First, in Section 2 the role of system parameters is discussed. Then in Section 3 the reservoir management examples are presented. The implications of the results are discussed and conclusions are drawn in Section 4.

\section{THE ROLE OF SYSTEM PARAMETERS}

Traditionally, system parameters are constant coefficients in a mathematical model structure. Since the introduction of modern systems theory with its state-space framework system parameters are also allowed to vary with time. Lee (1964) was among the first who pointed out the link between parameter and state estimation, so that the resemblance between the Kalman filter and the recursive least-squares parameter estimator became very clear. In this interpretation parameters are seen as time-varying unobserved states. Alternatively, after rewriting the solution of an LTI system in terms of a linear regression, states can be interpreted as linear regression parameters (e.g. Milanese and Belforte (1982)). In the early system theoretic literature on LQ theory the resemblance between optimal state estimation and optimal control has already been pointed out. In the eighties, and in particular within the framework of nonlinear control theory (see e.g. Nijmeijer and van der Schaft (1990)), inputs are

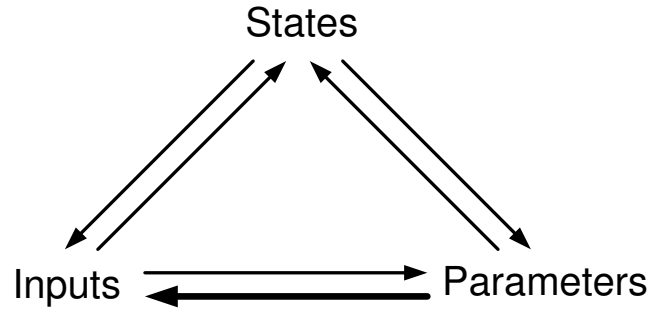

Fig. 1. The links between states, inputs and parameters.

really considered as states to arrive at a system representation that is affine in the control input. Moreover, in input estimation studies the unknown inputs are usually considered as states or equivalently as time-varying parameters (e.g. Corless and Tu (1998)). Fig. 1 summarizes the links between states, inputs and parameters.

Especially, the bold arrow in Fig. 1, indicating the interpretation of time-varying parameters as agents of control (Keesman (2002)), needs further attention. This link between parameters and control inputs will be further subject of the paper. For the interpretation of the results an additional view on system parameters is needed, i.e parameters as representatives of unknown sub-processes (see e.g. Whitehead (1979), Young (1984), Norton (1986) for environmental applications). Let us here illustrate this view by an ecological example.

Example 1. Consider the substrate consumption with Michaelis-Menten kinetics in a batch reactor or isolated lake. The substrate concentration is described by

$$
\frac{d S}{d t}=-\mu(S)
$$

with $\mu(S)=V_{\max } \frac{S}{K_{m}+S}$, the substrate degradation rate. Let $S_{0}=30, K_{m}=3$, and $V_{\max }=2$. Furthermore, over a period of 25 minutes, every minute the substrate concentration is sampled. The recursive estimation results, using the simplified model

$$
\frac{d S}{d t}=-\mu
$$

and the sampled data, are presented in Fig. 2 and 3. As can be seen from Fig. 3, the parameter $\mu$ is not a constant, indicating dependencies on other internal/external system variables. In fact, in the simplified model the parameter $\mu$ represents the 'hidden' Michaelis-Menten kinetics. In other words, via the recursively estimated parameters, either using a filter or smoothing algorithm, one may be able to discover unknown sub-processes using qualitative knowledge of the (bio)physics underlying the dynamics. 


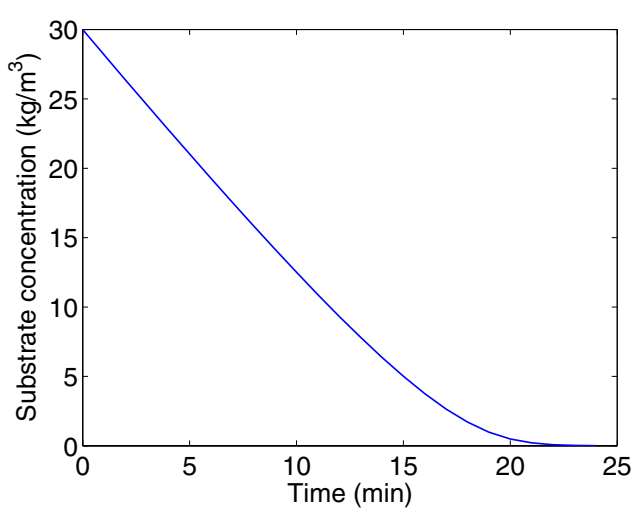

Fig. 2. Substrate concentration.

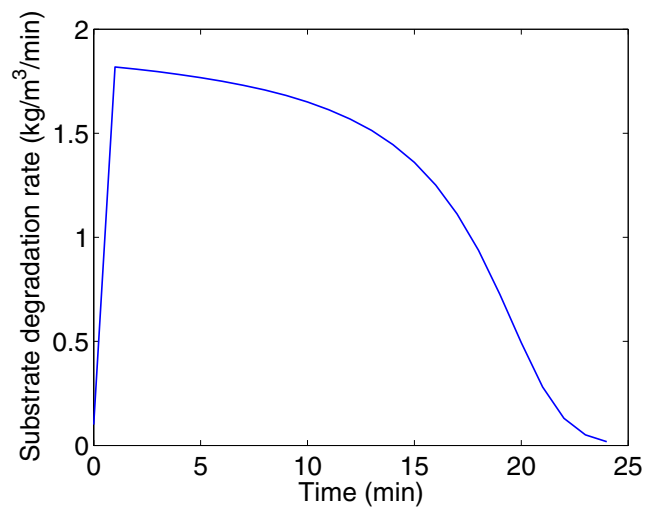

Fig. 3. Estimated substrate degradation rate.

Let us now further stress the role of time-varying parameters in management of environmental systems, potentially subject to system change. Consider, therefore, the feedback scheme of Fig. 4 with $\mathbf{P}$ the process and $\mathbf{C}$ the controller. It is wellknown that in the denominator of the transfer function of the LTI feedback system the product " $P C$ " always appears. Hence, instead of focussing on the design of $\mathbf{C}$ (as in traditional control theory and engineering) one could equally well focus on adjustment of $\mathbf{P}$, e.g. via parameter changes, or on both. This view on management and control of environmental systems can also be seen as an extension of the ideas on process design under feedback control via optimization, as initiated by e.g. Grossmann and Sargent (1978). Backward uncertainty propagation (Abusam et al. (2003)), where specific characteristics of an output probability density function, as for instance its tails, are related to regions in the parameter space, is another approach which shows links with the contents of this paper. As opposed to the previous two ideas, in our framework, however, the parameters are allowed to vary with time.

In conclusion, projective modeling opens the possibility to explore the effects, in terms of internal system changes, of user-defined, expected system outputs. In the next section this idea will be first illustrated on a real-world lake restoration

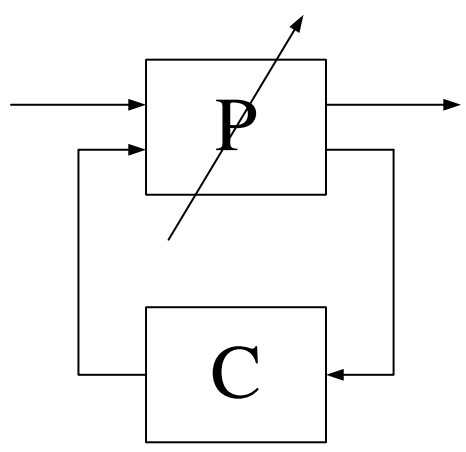

Fig. 4. Parameters as agents for control.

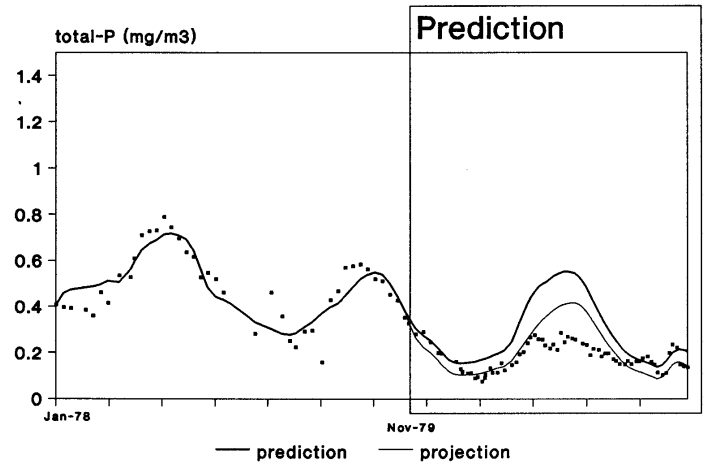

Fig. 5. Total phosphorus concentration in Lake Veluwe 1978-1980.

example and finally worked out in more detail on a simple reservoir management example.

\section{RESERVOIR MANAGEMENT EXAMPLES}

\subsection{Lake restoration}

The lake eutrophication problem in The Netherlands (see Keesman and van Straten, 1989; van Straten and Keesman, 1991) will be used here as an example of projective modeling. However, in all fairness, we have to admit that at that time the problem was approached from the opposite. Let us first follow the steps taken in that study and then apply a reverse way of reasoning. In our study the effective flushing strategy (Hosper and Meyer (1986)) had already been implemented. The ultimate goal of the restoration program was to limit the amount of phosphorus in one of the lakes up to a specific norm (approx. $300 \mathrm{mg} / \mathrm{m}^{3}$ ). Via sensitivity analysis and prior knowledge of the flushing strategy it was found that a reduction of the background concentration of phosphorus in the sediment (represented by a single model parameter $\beta$ ) to $25 \%$ of its original value in November 1979 gave rather good predictions, see Fig. 5. 
Let us now reverse the reasoning and start from the situation where we have to find appropriate control strategies. After defining the desired maximum phosphorus level, while remaining the periodicity of the phosphorus concentration, techniques to calculate the dominant parameter trajectories can be used. When it appears that (i) $\beta$ is a dominant parameter, and (ii) a jump in $\beta(t)$ suffices to reach the desired goal, we have to start thinking on the real implementation. One of the options is flushing, but dredging could be another option to reduce the concentration of phosphorus in the sediment. In the next step, when it appeared that reduction of the background phosphate concentration in the sediment via flushing is most effective in reaching the goal, a (weekly/monthly) flushing strategy has to be developed such that the background concentration in the sediment is reduced accordingly. Hence, a type of cascade control results, where $\beta$ is determined in the primary loop and the inflow of fresh water in the secondary loop (see Keesman (2002)).

In the next subsection the focus is on the calculation and physical interpretation of the appropriate dominant parameter trajectories.

\subsection{Reservoir capacity management}

Consider a reservoir with volume $V$ and area $A$. Furthermore, the outflow is proportional with the height $(h=V / A)$, so that the following LTI statespace model, describing the inflows and outflows, may result,

$$
\begin{aligned}
\frac{d h}{d t} & =-K h+\frac{u}{A} \\
y & =K h
\end{aligned}
$$

The input-output model, using the Euler forward difference method, in discrete-time is given by

$$
y_{k}=(1-K T) y_{k-1}+\frac{K T}{A} u_{k-1}
$$

Let $K=K^{o}=0.5 \mathrm{~d}^{-1}, A=A^{o}=25 \mathrm{~km}^{2}$, $y(0)=5 m d^{-1}$ and sample time $T=0.1 d$. Define the desired output $r(t)$ as given by Fig. 6 , where a sudden internal system change occurs at $t=20 d$ and let $u(t)=10^{6} \mathrm{~m}^{3} \mathrm{~d}^{-1}$, a constant inflow to the reservoir. Let us further explain the choice of $r(t)$. In Fig. 6, for $t \in[0,20], r(t)$ is a model response with parameters estimated from available experimental data, while for $t>$ $20 r(t)$ is exact and pre-specified by the user. Alternatively, for $t \in[0,20]$, the corresponding experimental data could have been used, but here we have chosen to utilize a smooth $r(t)$ to emphasize the effects of an internal system change.

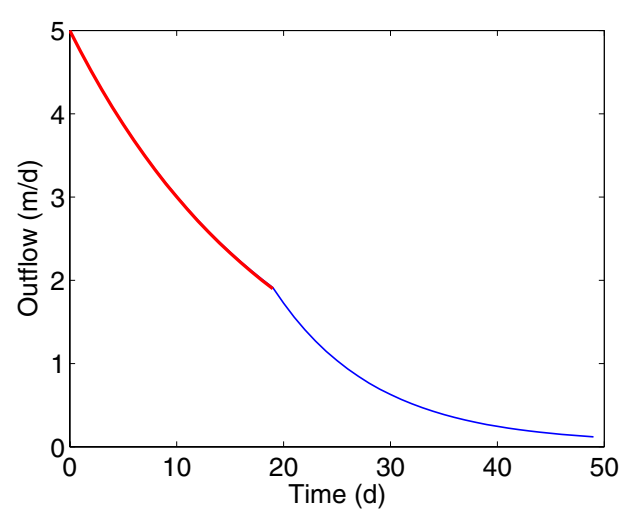

Fig. 6. Predefined outflow of reservoir.

In what follows, given the reference signal $r$ and input signal $u$, model predictive control (MPC) as well as recursive parameter estimation methods are used to find trajectories of $K$ and $A$ such that $y(t) \rightarrow r(t)$ for almost all $t$.

3.2.1. $M P C$ design In MPC design, without input weighting, the objective function to be minimized is usually given by

$$
J=\sum_{i \in \mathcal{P}}\left[r_{k+i / k}-y_{k+i / k}\right]^{2}
$$

with $\mathcal{P}$ the set of indices $i$ which correspond to coincidence points, in our case the sample points of $r(t)$. Let us start, in addition to (5), by evaluating the next model predictions, i.e. for $i \geq 1$

$$
\begin{aligned}
y_{k+1 / k} & =\left(1-K_{k} T\right) y_{k}+\frac{K_{k} T}{A} u_{k} \\
y_{k+2 / k} & =\left(1-K_{k+1} T\right) y_{k+1}+\frac{K_{k+1} T}{A} u_{k+1} \\
& \vdots \\
y_{k+i / k} & =y_{k} \prod_{n=1}^{i}\left(1-K_{k+i-n} T\right)+\cdots \\
& +u_{k} \prod_{n=1}^{i-1}\left(1-K_{k+i-n} T\right) \frac{K_{k} T}{A}+\cdots \\
& +u_{k+1} \prod_{n=1}^{i-2}\left(1-K_{k+i-n} T\right) \frac{K_{k+1} T}{A}+\cdots
\end{aligned}
$$

Notice that, as result of our choice to view $K$ as a control input, bilinear terms result. Consequently, initially it appears that a non-linear program has to be solved. However, if we consider each individual prediction step separately with $y_{k}=r_{k}$ and $K_{k}$ unbounded, then for this deterministic single input case a direct inversion approach is appropriate. Its solution with $A=A^{o}$ is given by: $K(t)=0.5,0 \leq t<20$ and $K(t)=1.0, t \geq 20$. If, on the contrary, we choose as control input $\alpha=\frac{1}{A}$, then the following predictions are obtained. 

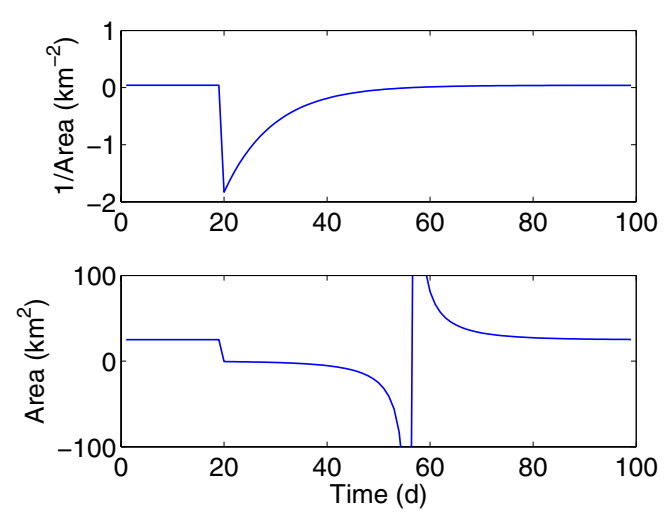

Fig. 7. Optimal inputs $\alpha(t)$ and $A(t)$.

$$
\begin{aligned}
y_{k+1 / k} & =(1-K T) y_{k}+K T u_{k} \alpha_{k} \\
y_{k+2 / k} & =(1-K T) y_{k+1}+K T u_{k+1} \alpha_{k+1} \\
& \vdots \\
y_{k+i / k} & =(1-K T)^{i} y_{k}+\cdots \\
& +(1-K T)^{i-1} K T u_{k} \alpha_{k}+\cdots \\
& +(1-K T) K T u_{k+i-2} \alpha_{k+i-2}+\cdots \\
& +K T u_{k+i-1} \alpha_{k+i-1}
\end{aligned}
$$

After some elementary algebraic manipulations, (8) can be written as a linear regression where its solution (see Fig. 7) can be easily found by Gauss elimination.

3.2.2. Recursive parameter estimation Alternatively, either $K$ or $A$, or both, can be calculated using recursive estimation techniques. For the reconstruction of $K(t)$ from the pre-defined output data (Fig. 6), we define the state-space model

$$
\begin{aligned}
K_{k+1} & =K_{k}+w_{k} \\
y_{k+1}-y_{k} & =\left[-T y_{k}+\frac{T u_{k}}{A}\right] K_{k}+v_{k}
\end{aligned}
$$

where $v_{k}$ and $w_{k}$ originate from zero mean white noise processes with (constant) covariance matrices $Q$ and $R$. In what follows $Q=1$, which allows sufficient parameter variations, and $R=0.001$ to express the presence of some model structure error due to e.g. errors in some of the other parameter values or approximate modeling errors. Hence, in the reconstruction of the parameter trajectories these errors must be taken into account. Fig. 8 shows the estimation results of $K$ for three different cases: $A<A^{o}, A=A^{o}, A>A^{o}$. Notice then that for $A=A^{\circ}$ similar results are found as in the MPC design case. For further interpretation of this result we note that for these choices of $Q$ and $R, Q \gg R$ and thus: $\lim _{k \rightarrow \infty} P_{k+1 / k} \rightarrow Q$ and $\lim _{k \rightarrow \infty} P_{k / k} \rightarrow R$, where $P_{k+1 / k}$ is the covariance matrix related to the predicted states and $P_{k / k}$ the covariance matrix related to the corrected

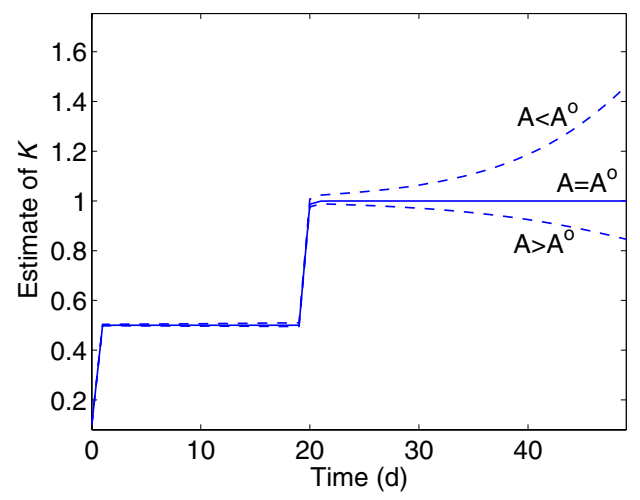

Fig. 8. Estimated trajectory of $K(t)$.

states. Under these noise assumptions analytical solutions to this estimation problem can also be found, but due to space limitations these are not shown here. A possible physical explanation of the trajectory of $K$ is that due to leakage (e.g. as a result of future erosion) extra water is extracted from the reservoir for $t>20 \mathrm{~d}$. In other words, we try to find changes in physical sub-processes that are represented by this single parameter in order to further understand the system's behavior under off-normal conditions.

For the reconstruction of $\alpha(t)$ from the pre-defined output data (Fig. 6), we define

$$
\begin{aligned}
\alpha_{k+1} & =\alpha_{k}+w_{k} \\
y_{k+1}-(1-K T) y_{k} & =K T u_{k} \alpha_{k}+v_{k}
\end{aligned}
$$

Again, $Q=1$ and $R=0.001$. The results for different values of $K$ are presented in Fig. 9. Notice from Fig. 9 that for some $K$ negative values of $\alpha_{k}$ result, so that $A_{k}$ shows some singular points. Clearly, in these cases a physical interpretation of $\alpha$ is not allowed. However, if we interpret $\alpha$ as a time-varying control input weight, then a negative value of $\alpha$ indicates that extra water should be taken from the reservoir, for instance by pumps, in order to meet the desired output. If, however, $K \geq 0.98: \hat{\alpha}_{k} \geq 0 \forall k \geq 0$. In these cases positive areas result, which implies a reservoir equipped with moving barriers, as in e.g lake IJsselmeer in The Netherlands.

The estimation results of both $K$ and $A$ are shown in Fig. 10. From Fig. 10 we notice that a systematic error in $\hat{K}$ appears $(\hat{K}=0.75$, while $K^{o}=1$ for $t \geq 20$ ). To compensate this, negative values of $\alpha$ appear in the period $t \in[20,40]$, which implies extra extraction of water.

\section{DISCUSSION AND CONCLUSIONS}

Clearly, other scenarios can be evaluated, as for instance a sudden jump in the outflow leading to a sudden reduction of the estimates of 

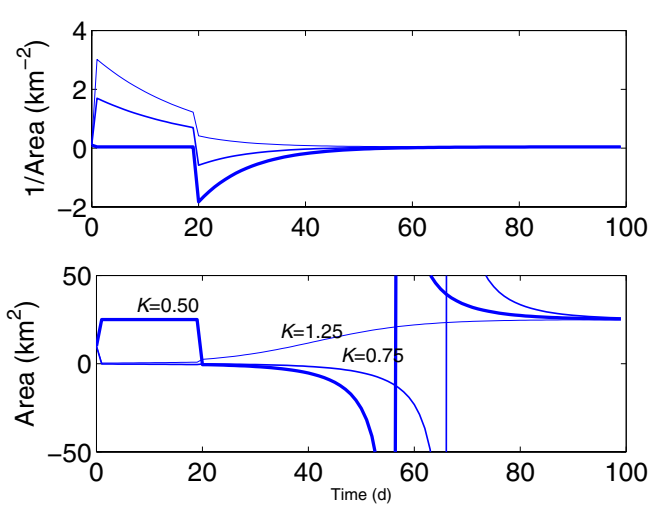

Fig. 9. Estimated trajectory of $1 / A(t)$ and $A(t)$.
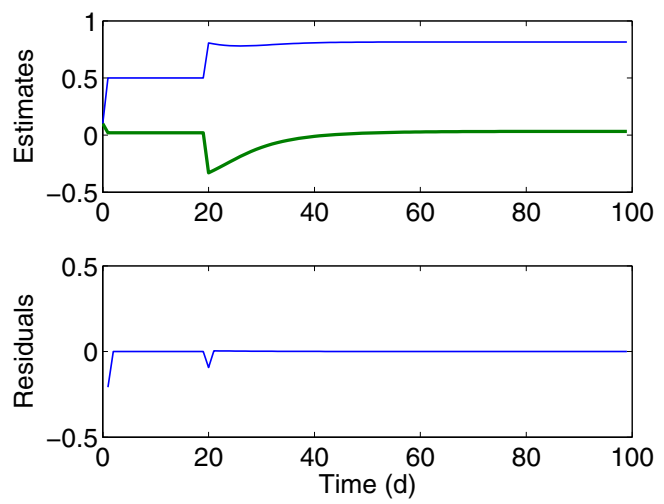

Fig. 10. Estimated trajectories of $K$ and $A(t)$ and residuals.

$K$ or $A$. Apart from the determination of the most dominant (identifiable) parameter(s) and the reconstruction of the parameter trajectories, in practice the crucial step remains the (bio)physical/chemical interpretation of the estimation results.

The following concluding remarks can be made:

- the interpretation of parameters as agents for control is very valuable in modeling future system changes.

- recursive estimation techniques provide a tool to reconstruct parameter trajectories from predefined future output behavior.

- in the projective modeling context the view of parameters representing underlying subprocesses helps to find new control strategies or "unexpected" system disturbances

- future research will focus on projective modeling under uncertainty.

\section{REFERENCES}

A. Abusam, K. J. Keesman, and G. van Straten. Forward and backward uncertainty propagation: an oxidation ditch modelling example. Water Research, 37(2):429-435, 2003.
A.C. Antoulas and J.C. Willems. A behavioral approach to linear exact modeling. IEEE Trans. AC, 38(12):1776-1802, 1993.

M.B. Beck. Environmental Foresight and Models: a manifesto. Elsevier, Amsterdam, 2002.

M. Corless and J. Tu. State and input estimation for a class of uncertain systems. Automatica, 34(6):757-764, 1998.

I.E. Grossmann and R.W.H. Sargent. Optimum design of chemical plants with uncertain parameters. AIChE J., 24:1021-1034, 1978.

H. Hosper and M. Meyer. Control of phosphorus loading and flushing as restoration methods for Lake Veluwe, The Netherlands. Hydrobiological Bulletin, 20:183-194, 1986.

K. J. Keesman. Parametric change as the agent of control. In: Environmental Foresight and Models: a manifesto, Ed. M.B. Beck, pages 415423, 2002.

K. J. Keesman and G. van Straten. Identification and prediction propagation of uncertainty in models with bounded noise. Int. J. Control, 49:2259-2269, 1987.

A.V. Kryazhimskii and M.B. Beck. Identifying the inclination of a system towards a terminal state from current observations. In: Environmental Foresight and Models: a manifesto, Ed. M.B. Beck, pages 425-450, 2002.

A.B. Kurzhanski and P. Varaiya. Ellipsoidal Techniques for Reachability Analysis. Lecture Notes in Computer Science, Volume 1790, SpringerVerlag GmbH, 2000.

R.C.K. Lee. Optimal Identification, Estimation and Control. MIT Press, Cambr., MA, 1964.

L. Ljung. System Identification - Theory for the User. Prentice Hall, 1987.

M. Milanese and G. Belforte. Estimation theory and uncertainty intervals evaluation in presence of unknown but bounded errors. IEEE Trans. Automat. Control, AC 27(2):408-414, 1982.

H. Nijmeijer and A.J. van der Schaft. Nonlinear dynamical control systems. Springer, 1990.

J. P. Norton. An Introduction to Identification. Academic Press, 1986.

C.P. Ormell. A new naturalistic style of mathematics. Phys. Educ., 12:257-260, 1977.

J.L. Tiwari and J.E. Hobbie. Random differential equations as models of ecosystems: Monte carlo simulation approach. Math. Biosci., 28:25-44, 1976.

G. van Straten and K. J. Keesman. Uncertainty propagation and speculation in projective forecasts of environmental change - a lake eutrophication example. J. Forecast., 10:163-190, 1991.

P.G. Whitehead. Applications of recursive estimation techniques to time variable hydrological systems. J. Hydrology, 40:1-16, 1979.

P. C. Young. Recursive Estimation and TimeSeries Analysis: An Introduction. Springer, 1984. 